\title{
A relação entre a percepção de competência física, índice de massa corporal e competência efectiva em jovens praticantes de basquetebol
}

Sandro Ferreira, Hélder M. Fernandes, José Vasconscelos-Raposo

FCT - Centro de Estudos em Desenvolvimento Humano, Actividade Física e Saúde

Ferreira, S.; Fernandes, H; Vasconcelos-Raposo, J.; A relação entre a percepção de competência física, índice de massa corporal e competência efectiva em jovens praticantes de basquetebol. Motricidade 3(3): 57-72

\section{Resumo}

Este estudo tem como objectivos: (i) explorar as relações entre o índice de massa corporal (IMC) e a percepção de competência física (PCF e/ou a Competência Efectiva em Basquetebol CEB; (ii) perceber a relação entre a PCF e a CEB, e (ii) determinar quais são as fontes de informação que os jovens utilizam para determinar a percepção de competência física.

Para tal, foi aplicado a 156 jovens estudantes com idades compreendidas entre os 12 e 18 anos $(\mathrm{M}=14.36 ; \mathrm{SD}=1.76)$, a subescala de percepção de competência física (SPCF), a escala de informação de competência física (PCIS) e um protocolo para a competência efectiva em Basquetebol.

Os principais resultados revelaram: (i) diferenças estatisticamente significativas entre sexos para o IMC, apresentando os rapazes valores médios mais elevados; (ii) média superior dos rapazes superior às raparigas, para a PCF e CEB; (iii) diferenças estatisticamente significativas entre sexos para os seguintes factores do PCIS: negativismo pré-competitivo e atracção pelo Desporto; e, (iv) diferenças estatisticamente significativas entre as diferentes faixas etárias para o factor avaliação dos pais.

Palavras chave: Percepção de competência física, IMC, Basquetebol.

\section{Abstract}

Relatioships between physical aptitude perception, body mass index and true aptitude in young basketball players

The purposes of this study were: (i) explore the relationship between Body Mass Index (BMI) and Perceived Physical Competence (PPC), and/or Effective Basketball Competence (ECB); (ii) understand the relationship between PPC and ECB; and, (iii) establish the sources of information that the young use to define the PPC.

A sample of 156 physical education students, with ages ranging from 12 to 18 years $(M=14.36$; $\mathrm{SD}=1.76)$, completed the perception of physical competence subscale (PPCS) and the physical competence source's subscale (PCSS). They also realized a protocol to measure effective competence in Basketball.

The main results revealed: (i) significant differences between boys and girls, while comparing BMI, with higher mean scores for boys; (ii) higher mean scores of PPC and BEC for boys; (iii) significant differences for the following constructs of the PCSS: pre-competition anxiety and sport attraction; and, (iv) significant differences between different groups of ages, for parent's evaluation factor.

Keywords: Physical aptitude perception, body mass index, Basketball 


\section{Introdução}

Acredita-se que o desporto tem um papel importante na socialização dos jovens, uma vez que o contacto social permite que eles adquiram e desenvolvam valores sociais que são defendidos por esta ${ }^{53}$. O Desporto é um contexto clássico de "realização" pessoal. O indivíduo ou as equipas confrontam-se para alcançar um objectivo, ou confrontam-se para melhorar as suas prestações (podendo ser avaliado em termos de sucesso ou fracasso). Assim, os indivíduos ou a equipa são os responsáveis pelo resultado e há um certo nível de desafio que é percepcionado.

Allen e Howe ${ }^{1}$ referem que a teoria da competência motivacional de Harter $^{28,30}$ é ajustada à explicação do comportamento dos jovens no Desporto, dado que o aspecto central da teoria é a percepção individual de competência num determinado domínio. Desta forma, quando um jovem acredita que é "competente" numa actividade, tende a estar mais predisposto a se envolver nessa actividade ou numa actividade semelhante, no futuro. Ser competente no Desporto e nas habilidades desportivas, é muito importante para os jovens, em particular para os rapazes ${ }^{53}$.

Dado que os rapazes sempre tiveram mais liberdade do que as raparigas para permanecerem na rua e jogarem a brincadeiras habituais como o apanha, escondidas, ou à bola, o envolvimento das mulheres a um conjunto de profissões que tradicionalmente são de homens (como no exército ou no Desporto), veio modificar a forma como esta se percepciona em diversos domínios, nomeadamente no domínio físico.

Atendendo a este facto, aquilo a que nos propomos é examinar simultaneamente em diferentes faixas etárias e entre sexos: (i) as relações entre Índice de Massa Corporal (IMC) e a Percepção de Competência Física (PCF) e/ou a Competência Efectiva em Basquetebol (CEB); (ii) a relação entre PCF e a CEB; e, (iii) determinar quais são as fontes de informação que os jovens utilizam para determinar a sua PCF.
A competência tem vários significados na Psicologia, indo desde um processo motivacional, até um comportamento efectivo na simples tarefa de viver ${ }^{22,25,46,60}$. A competência pertence a um conjunto de construtos que incluem o ego ${ }^{6,34,44}$, a auto-eficácia ${ }^{3,72}$ a inteligência e o comportamento inteligente ${ }^{13,58,61}$, a competência pré-mórbida ${ }^{24,52,75}$ e o desenvolvimento de tarefas ${ }^{57}$.

Para Butt ${ }^{12}$, a competência é a interacção efectiva do indivíduo com o meio ambiente. Para este, existem 5 grandes categorias do comportamento humano, que podem expressar a competência: (i) competência física, (ii) competência intelectual, (iii) competência emocional, (iv) competência social e (v) competência espiritual ou existencial. Não se pode esperar que um indivíduo tenha os mesmos níveis de competência nas 5 categorias. No entanto, o indivíduo terá experiências de competência nessas 5 categorias.

Para alguns autores ${ }^{1,12,62}$ a teoria da competência motivacional de Harter ${ }^{28}$ é ajustada à explicação do comportamento dos jovens no Desporto. Inicialmente, esta teoria foi conceptualizada por White ${ }^{72}$, sendo mais tarde operacionalizada por $\mathrm{Harter}^{28,30}$.

A tese de White refere que os indivíduos estão motivados para lidar com competência dentro do seu domínio social e físico, e por isso, comprometendo-se em tentar a mestria. Se essas tentativas para a mestria resultarem em sucesso ou prestações competentes, serão vivenciados sentimentos que manterão a motivação intrínseca do indivíduo ${ }^{63}$. Após 20 anos de dados empíricos que suportaram esta teoria, Harter $^{28}$ forneceu uma extensão refinada da formulação original, que incluía várias componentes que influenciam a orientação motivacional do indivíduo.

$\mathrm{O}$ aspecto central da teoria motivacional de Harter é a percepção individual de competência num determinado domínio ${ }^{1}$, pelo que este modelo de competência motivacional prediz que a competência percepcionada de um domínio de excelência, será crítica para uma subsequente motivação ${ }^{62}$. 
$\mathrm{Na}$ sua teoria, Harter argumenta que os indivíduos motivados intrinsecamente são aqueles que demonstram a sua habilidade na tentativa de mestria, usando critérios internos e objectivos relativos à mestria para avaliar o sucesso e a sua competência percepcionada ${ }^{63}$. Por outro lado, os indivíduos motivados extrinsecamente, evitam tentar a mestria, porque desta forma, minimizam a probabilidade de demonstrar uma baixa competência. Dependem de critérios externos para avaliar a sua prestação (suporte social) e possuem uma baixa percepção da sua competência, pelo que vivenciam situações de negativismo.

Quanto a estudos respeitantes à competência percepcionada e aos motivos de participação no Desporto, é possível retirar as seguintes conclusões: (i) os jovens com elevadas percepções de competência, estão mais motivados para desenvolver as suas habilidades, enquanto os que possuem uma elevada percepção de competência social, estão motivados por razões de afiliação ${ }^{40}$; (ii) os participantes no Desporto apresentam uma maior percepção da sua competência física, do que os não participantes ou desistentes ${ }^{21,41,54,63}$; (iii) uma elevada percepção de competência física, leva os jovens a esperar mais sucesso no futuro, prolongando o seu envolvimento na actividade $^{54}$; e (iv) os atletas com uma elevada percepção de competência física, consideraram o desenvolvimento das suas habilidades, a afiliação, e o divertimento, como razões mais importantes para a sua participação no Desporto ${ }^{55}$.

Harter referiu que um ponto crítico que se adicionou à teoria de White, envolve a história da socialização do jovem. Desta forma, a influência dos outros no reforço da motivação intrínseca ou extrínseca, através do julgamento pessoal da competência e do controlo, é especialmente crítico durante a infância e adolescência ${ }^{63}$. Assim, um aumento da motivação intrínseca levaria a um aumento da competência percepcionada ${ }^{1}$, enquanto uma percepção negativa, teria como consequência uma diminuição da competência percepcionada, o que por sua vez levaria a um aumento da negativismo e uma diminuição da possibilidade desse jovem se comprometer no futuro em actividades desportivas. Tal como Weiss e Duncan ${ }^{64}$ referem, a competência física está relacionada significativamente com a crença da competência dos jovens, na aceitação social.

Por sua vez, o modelo de Harter também sugere que há uma relação entre o sucesso, a percepção de sucesso, a competência, o controlo, o afecto e a orientação motivacional, pelo que originou um conjunto de estudos que corroboram esta ideia ${ }^{63}$. Num outro trabalho ${ }^{65}$ foi revelado que raparigas sub-avaliadas na sua percepção de competência, tinham valores baixos de motivação intrínseca e uma elevada negativismo, ao contrário das raparigas sobre-avaliadas, pelo que as primeiras e mais generalizadamente, os jovens nesta situação, são sérios candidatos a desistirem mais cedo da prática desportiva.

Uma importante componente no modelo de Harter, é a preferência por fontes de informação que reforcem as tentativas de mestria e de sucesso, assim como o estabelecer de objectivos. O indivíduo que é motivado intrinsecamente interioriza um sistema de auto-recompensa e define um conjunto de objectivos de mestria como resultado positivo das experiências de socialização. Contudo, o indivíduo que é motivado extrinsecamente depende de uma aprovação e reforço externo, para julgar a sua habilidade e para definir objectivos.

A investigação nesta área tem evidenciado que existem relações entre a idade e as fontes de informação para julgar a competência física. Especificamente, os jovens com 8 e 9 anos usam o resultado dos jogos e os feedbacks dos adultos, dado que é algo muito concreto e facilmente interpretável. Ao contrário, os jovens de 10 a 14 anos, utilizam a comparação com os colegas, para avaliar a sua competência. 
Nicholls ${ }^{48}$ construiu a noção de orientação motivacional para a habilidade e para a tarefa. Ambas as orientações se relacionam com o conceito de habilidade ${ }^{18,47,73,74}$. No entanto, este conceito foi visto como tendo diferentes significados para os indivíduos orientados para a habilidade e para a tarefa.

Quando um indivíduo está envolvido para a tarefa, uma maior aprendizagem resulta no aumento da competência percepcionada e a dificuldade da tarefa e o julgamento da habilidade são auto-referenciados ${ }^{59}$. Um atleta que está orientado para a tarefa, tende a processar a sua habilidade em termos de critérios pessoais ${ }^{47}$. Porque um grande esforço despendido resulta numa maior aprendizagem, as tarefas difíceis e que necessitam de grande esforço, dão oportunidade para aprender e vivenciar sentimentos de competência.

A investigação suportou a teoria de $\mathrm{Nicholls}^{48}$, quando referiu que os indivíduos com uma orientação para a habilidade/ego, acreditavam que eram bons atletas, quando a sua prestação era tão boa ou melhor que a dos outros, ou melhor, mas despendiam com isso menos esforço. Contudo, os indivíduos com uma orientação para a tarefa, acreditavam que eram bons atletas quando trabalhavam, aprendiam e melhoravam.

Quando falamos de um envolvimento para o ego, esta orientação está relacionada com fontes de informação associadas à comparação social, tais como o resultado final de um jogo, avaliação dos outros e a comparação com os colegas ${ }^{74}$. Em contraste, a orientação para a tarefa, está relacionada com fontes de informação como a aprendizagem e o esforço.

A variação da motivação intrínseca está associada segundo Nicholls $^{50}$ e Dweck ${ }^{20}$ à orientação cognitiva. Presume-se que um envolvimento para a tarefa estará positivamente associado a uma motivação intrínseca, enquanto que um envolvimento para o ego, corresponderá a uma diminuição dessa mesma motivação intrínseca.

Desta forma, quanto mais os indivíduos estão motivados intrinsecamente, mais eles se percepcionam como competentes ${ }^{62}$, assim como as crianças motivadas intrinsecamente, num determinado domínio, percepcionar-se-ão como competentes.

O padrão geral de crescimento pós-natal é muito semelhante de indivíduo para indivíduo. Mas há uma variabilidade individual no que respeita à velocidade de crescimento em diferentes idades, considerando o corpo como um todo ou apenas partes do corpo ${ }^{45}$. Estes mesmos autores, referem que cada medição corporal permite obter informações específicas para entendermos o crescimento.

O peso e a altura, são as suas medições mais usadas para estudar o crescimento, podendo ser relacionadas. Esta relação, por sua vez, é muito utilizada em pré-adolescentes. Normalmente, as crianças com problemas nutricionais têm um baixo rácio peso/altura. Pelo contrário, as crianças inactivas e quase sempre bem nutridas, têm elevados rácios peso/altura.

O índice de massa corporal (IMC) representa o quociente entre o peso (em quilogramas) e o quadrado da altura (em metros). Este índice é de grande importância prática: (i) tem uma correlação com a mortalidade e morbilidade gerais, e relação com diversas patologias, permitindo uma estratificação de riscos; (ii) permite a variação individual, dentro de cada zona de risco; e, (iii) correlaciona-se com a quantidade de massa gorda, que na população geral pode ser predita por ele, quer em indivíduos normoponderais, quer em obesos, correlação esta que ainda melhora, se for integrada com o sexo e a idade, mediante equações apropriadas.

Barata ${ }^{4}$ apresenta, para homens, grupos de risco entre os 25.1 e $30.0 \mathrm{~kg} / \mathrm{m}^{2}$. Também foi definido que como obesidade um IMC acima de $30.0 \mathrm{~kg} / \mathrm{m}^{2}$. 


\section{Metodologia}

\section{Amostra}

A amostra foi constituída por 156 jovens estudantes sendo 76 do sexo masculino e 80 do sexo feminino. Os estudantes tinham idades compreendidas entre os 12 e os 18 anos $(\mathrm{M}=$ $=14.36 ; \mathrm{SD}=1.76)$. A amostra englobou estudantes do $7 .^{\circ}, 10 .^{\circ}$ e $11 .^{\circ}$ anos de escolaridade.

Este grupo de idade foi escolhido, porque a investigação tem demonstrado que os jovens com menos de 12 anos estão menos aptos a diferenciar o esforço da habilidade/competência, e por isso, são menos capazes de discernir a sua própria competência.

\section{Procedimentos}

Quanto aos instrumentos, os alunos responderam numa sala de aulas, à Subescala de Percepção de Competência Física (SPCF) de Harter ${ }^{32}$, traduzida para português e à Escala de Informação de Competência Física (PCIS) ${ }^{70}$, que também foi traduzida para português. Estes dois questionários possibilitam ter acesso à percepção que cada aluno tem da sua competência (SPCF) $\mathrm{e}$ as fontes de informação que os alunos utilizam para definir essa competência (PCIS). O primeiro é composto por 7 itens, enquanto o segundo é composto por 33 itens. Para ambos, foi utilizada uma escala tipo Likert de 5 pontos, em que 1 corresponde a discordo completamente e 5 a concordo completamente.

Depois de responderem aos questionários, foram medidas a altura e o peso para posterior cálculo do IMC. Para a altura foi colocada uma fita métrica na parede. Os indivíduos tinham que se colocar na posição anatómica de estudo e só depois seria registada a medição. Para a medição do peso, os indivíduos foram medidos sem calçado e apenas com uma t-shirt e calções ${ }^{45}$, com uma balança Salter Electronic. Ambas as medições foram recolhidas entre as $9 \mathrm{~h} 30 \mathrm{~m}$ e as $12 \mathrm{~h} 30 \mathrm{~m}$ no período da manhã e das $16 \mathrm{~h} 30 \mathrm{~m}$ às $18 \mathrm{~h} 30 \mathrm{~m}$, no período da tarde.
Para avaliar a competência efectiva em Basquetebol, os alunos começaram por correr 5 minutos à volta de $1 / 3$ de um pavilhão desportivo. Foi-lhes dito que o objectivo não seria chegar em primeiro lugar, mas sim, aumentar a temperatura corporal, pelo que seria importante não pararem. Depois da corrida contínua, todos os alunos tiveram 10 minutos de treino de lances-livres (LL). Os alunos com idade inferior a 14 anos, lançaram de 4 metros, enquanto os alunos com idade superior a 14 anos, lançaram de 4.70 metros. Foram colocadas 2 tabelas móveis com uma altura de $3.05 \mathrm{mt}$, paralelas uma à outra. Em cada uma, ficou colocada uma fila com 5 bolas. $\mathrm{O}$ aluno lançava, recolhia o seu próprio ressalto e passava ao primeiro colega da fila que não tinha bola. Houve uma demonstração inicial e foi-lhes dito para não brincarem na fila aquando do LL, porque significaria que teriam menos lançamentos que os seus colegas da outra fila. Em cada tabela e em simultâneo, cada aluno realizou 10 LL, sendo essa percentagem registada no próprio questionário pelos professores presentes.

As variáveis dependentes que suportam a realização do nosso estudo, são o IMC, a PCF, a CEB e as fontes de informação onde os indivíduos vão retirar a sua percepção de competência.

\section{Estatística}

Nos procedimentos estatísticos, foram calculadas as estatísticas descritivas (média e desvio padrão), para todas as variáveis. Foi realizada uma validação interna de cada factor do PCIS, obtendo-se o valor do $\alpha$ de Cronbach, que tem como expressão máxima de validade interna o valor de 1. Também foi realizada uma análise multivariada (MANOVA), afim de testar as diferenças entre sexos e grupos etários, para todas as variáveis dependentes. Por fim, foi efectuada uma correlação produto-momento de Pearson (r) entre a PCF e a CEB. 
A relação entre a percepção de competência física, índice de massa corporal e competência efectiva em jovens praticantes de basquetebol

Sandro Ferreira, Hélder Fernandes, José Vasconcelos-Raposo

\section{Resultados}

A apresentação dos dados será feita com a seguinte ordem: (i) análise dos resultados do IMC; (ii) análise dos resultados da SPCF; (iii) análise dos resultados da CEB; e, (iv) análise dos resultados do PCIS.

Assim, os seguintes quadros apresentam-nos a média e o desvio padrão do IMC, relativamente ao sexo (quadro 1) e à idade (quadro 2), respectivamente.

Quadro 1: Estatística descritiva do IMC por sexo.

\begin{tabular}{ccccc} 
& Sexo & N & Média & $\begin{array}{c}\text { Desvio } \\
\text { Padrão }\end{array}$ \\
\cline { 2 - 5 } IMC & Masculino & 76 & 20.99 & 3.34 \\
& Feminino & 80 & 20.73 & 2.90 \\
\hline
\end{tabular}

Verificamos que os rapazes obtiveram uma média de $20.99 \pm 3.34$ e as raparigas de $20.73 \pm 2.90$. Quando analisando o IMC por idades, constatamos que são as idades de 14, 15, 16 e 17 anos que apresentam os valores médios mais elevados.

Quadro 2: Estatística descritiva do IMC por idades.

\begin{tabular}{ccccc} 
& Idade & N & Média & $\begin{array}{c}\text { Desvio } \\
\text { Padrão }\end{array}$ \\
\hline \multirow{4}{*}{ IMC } & 12 & 30 & 19.44 & 3.48 \\
& 13 & 25 & 20.63 & 3.56 \\
& 14 & 29 & 21.35 & 3.32 \\
& 15 & 31 & 21.51 & 2.67 \\
& 16 & 22 & 21.61 & 1.90 \\
& 17 & 9 & 21.21 & 2.25 \\
& 18 & 10 & 20.25 & 3.52 \\
\hline
\end{tabular}

A análise multivariada, revelou-nos diferenças estatisticamente significativas entre as idades, para esta variável $(\mathrm{F}=4.031 ; \mathrm{p}<0.046)$.

Relativamente à PCF, os rapazes apresentam uma média superior à das raparigas. Também na competência efectiva no Basquetebol, os rapazes obtiveram valores médios mais elevados (quadro 3).

Quadro 3:

Estatística descritiva da PCF e da CEB por sexo

\begin{tabular}{ccccc}
\multirow{2}{*}{ PCF } & Sexo & N & Média & $\begin{array}{c}\text { Desvio } \\
\text { Padrão }\end{array}$ \\
\cline { 2 - 5 } & Masculino & 76 & 25.01 & 3.96 \\
& Feminino & 80 & 22.00 & 3.79 \\
\hline \multirow{2}{*}{ CEB } & Masculino & 76 & 25.13 & 16.85 \\
& Feminino & 80 & 13.50 & 11.26 \\
\hline
\end{tabular}

$\mathrm{Na}$ análise da PCF por idades, verificamos que são as idades de 12, 13 e 18 anos, a apresentarem os valores médios mais elevados (quadro 4).

Quadro 4: Estatística descritiva da PCF por idades.

\begin{tabular}{ccccc} 
& Idade & N & Média & $\begin{array}{c}\text { Desvio } \\
\text { Padrão }\end{array}$ \\
\hline \multirow{4}{*}{ PCF } & 30 & 25.20 & 3.60 \\
& 13 & 25 & 24.44 & 4.50 \\
& 14 & 29 & 23.00 & 2.98 \\
& 15 & 31 & 22.71 & 5.18 \\
& 16 & 22 & 22.27 & 4.61 \\
& 17 & 9 & 22.67 & 3.24 \\
18 & 10 & 23.30 & 2.58 \\
\hline
\end{tabular}


No quadro seguinte, podemos observar que são os jovens de 16 anos que apresentam o valor médio mais alto de CEB, seguidos pelos jovens de 12 anos (quadro 5).

A correlação de Pearson revelou-nos uma relação baixa, mas significativa entre a PCF e a CEB ( $r=0.182 ; \mathrm{p}<0.05)$.

A análise multivariada (MANOVA) revelou-nos uma diferença estatisticamente significativa entre os sexos, quer para a PCF $(\mathrm{F}=$ $=21.216$; $\mathrm{p}<0.000)$, quer para a $\mathrm{CEB}(\mathrm{F}=24.484$; $\mathrm{p}<0.000$ ) (quadro 6).
Quadro 5: Estatística descritiva da CEB por idades.

\begin{tabular}{ccccc} 
& Idade & N & Média & $\begin{array}{c}\text { Desvio } \\
\text { Padrão }\end{array}$ \\
\hline 12 & 30 & 24.33 & 16.54 \\
& 13 & 25 & 16.00 & 10.00 \\
CEB & 14 & 29 & 18.62 & 17.26 \\
& 15 & 31 & 16.13 & 12.83 \\
& 16 & 22 & 25.45 & 18.19 \\
& 17 & 9 & 10.00 & 10.00 \\
& 18 & 10 & 17.00 & 15.67 \\
\hline
\end{tabular}

Quadro 6: Valores da análise multivariada por sexo.

\begin{tabular}{lcccc} 
Variável & Soma quadrados & Graus Liberdade & F & Sig. \\
\hline PCF & 312.465 & 1 & 21.216 & 0.000 \\
CEB & 5006.219 & 1 & 24.484 & 0.000 \\
\hline
\end{tabular}

O mesmo tipo de análise, revelou-nos uma diferença estatisticamente significativa na PCF, relativamente às diferentes idades da amostra $(\mathrm{F}=4.031 ; \mathrm{p}<0.046)$.

O PCIS permite-nos saber quais as fontes de informação que os jovens utilizam para avaliar a sua competência física. No quadro 7, estão expostos o valores de $\alpha$ de Cronbach para os estudos de Weiss, Horn e Ebbeck $^{70}$ e para o nosso estudo. No primeiro, os quatro primeiros factores obtiveram um valor superior a 0.70 . Por isso, foram considerados indicadores fiáveis para fontes de informação relativas à PCF. No nosso estudo, só foram encontrados valores de $\alpha$ de Cronbach superiores a 0.70 na comparação social e avaliação dos pais. Nas restantes escalas (comportamentos auto-referenciados e negativismo pré-competitivo) os valores são inferiores, sendo quase iguais para o factor atracção pelo Desporto e bastante mais baixos na facilidade em aprender habilidades (quadro 7).

Apesar destes valores de consistência interna, quisemos determinar se existiam diferenças estatisticamente significativas entre os sexos e entre as idades, para estes seis factores. 
A relação entre a percepção de competência física, índice de massa corporal e competência efectiva em jovens praticantes de basquetebol

Sandro Ferreira, Hélder Fernandes, José Vasconcelos-Raposo

Quadro 7: Valores $\alpha$ de Cronbach do estudo de Weiss, Horn e Ebbeck (1997) e do presente estudo (2001).

\begin{tabular}{lcc} 
Factores & $\begin{array}{c}\text { Weiss,Horn e Ebbeck } \\
(1997)\end{array}$ & $\begin{array}{c}\text { Ferreira, Raposo } \\
\text { e Fernandes (2001) }\end{array}$ \\
\hline Comparação Social & 0.88 & 0.79 \\
Comportamentos auto-referenciados & 0.84 & 0.62 \\
Avaliação dos pais & 0.80 & 0.70 \\
Negativismo pré-competitivo & 0.72 & 0.68 \\
Atracção pelo Desporto & 0.68 & 0.69 \\
Facilidade em aprender habilidades & 0.61 & 0.27 \\
\hline
\end{tabular}

O quadro seguinte, mostra-nos a média e desvio padrão dos 6 factores diferenciados por sexo. Os rapazes apresentam valores superiores aos das raparigas, em 4 do total de 6 factores. Como tal, para os factores comparação social, comportamentos auto-referenciados, avaliação dos pais e atracção pelo Desporto, o valor médio dos rapazes é superior ao das raparigas. Por sua vez, para os factores negativismo pré-competitivo e facilidade em aprender habilidades, o valor médio das raparigas é superior ao dos rapazes (quadro 8).

Quadro 8: Média e desvio padrão para os 6 factores do PCIS por sexo.

\begin{tabular}{lcccc} 
Factores & Sexo & N & Média & Desvio Padrão \\
\hline Comparação Social & Masculino & 76 & 42.63 & 6.97 \\
& Feminino & 80 & 40.19 & 7.06 \\
Comportamentos & Masculino & 76 & 33.55 & 3.75 \\
auto-referenciados & Feminino & 80 & 33.06 & 3.57 \\
Avaliação dos pais & Masculino & 76 & 10.22 & 2.54 \\
& Feminino & 80 & 9.99 & 2.39 \\
Negativismo pré-competitivo & Masculino & 76 & 9.03 & 2.89 \\
& Feminino & 80 & 10.20 & 2.46 \\
Atracção pelo Desporto & Masculino & 76 & 8.89 & 1.41 \\
& Feminino & 80 & 8.38 & 1.41 \\
Facilidade em & Masculino & 76 & 10.12 & 1.90 \\
aprender habilidades & Feminino & 80 & 10.41 & 1.86 \\
\hline
\end{tabular}

A análise multivariada entre sexos para os factores do PCIS, revelou-nos diferenças estatisticamente significativas para os factores negativismo pré-competitivo ( $\mathrm{F}=6.522$; $\mathrm{p}<0.012)$ e atracção pelo Desporto $(\mathrm{F}=5.065$; $\mathrm{p}<0.026$ ) (quadro 9). 
Quadro 9: Valores de F da análise multivariada por sexo.

\begin{tabular}{|c|c|c|c|c|}
\hline Variável & Soma quadrados & Graus Liberdade & $\mathbf{F}$ & Sig. \\
\hline $\begin{array}{l}\text { Negativismo } \\
\text { Pré-competitivo }\end{array}$ & 46.832 & 1 & 6.552 & 0.012 \\
\hline $\begin{array}{l}\text { Atracção } \\
\text { pelo Desporto }\end{array}$ & 10.194 & 1 & 5.065 & 0.026 \\
\hline
\end{tabular}

Quando analisamos estes 6 factores por idades, constatamos apenas uma diferença estatisticamente significativa para o factor avaliação dos pais $(\mathrm{F}=11.151 ; \mathrm{p}<0.001)$. Desta forma, neste mesmo factor os valores médios mais elevados foram para as idades de 13, 12 e 18 anos, respectivamente (quadro 10).

Quadro 10: Estatística descritiva do factor Comparação Social por idades.

\begin{tabular}{lcccc} 
& Idade & N & Média & $\begin{array}{c}\text { Desvio } \\
\text { Padrão }\end{array}$ \\
\cline { 2 - 5 } & 12 & 30 & 43.00 & 6.72 \\
& 13 & 25 & 43.36 & 7.13 \\
Comp. & 14 & 29 & 40.76 & 5.87 \\
Social & 15 & 31 & 39.90 & 8.09 \\
& 16 & 22 & 39.50 & 8.88 \\
& 17 & 9 & 40.89 & 5.06 \\
& 18 & 10 & 42.50 & 4.09 \\
\hline
\end{tabular}

No factor comportamentos auto-referenciados, os jovens com 15 e 16 anos de idade, foram os que obtiveram valores médios mais elevados (quadro 11).

Quadro 11: Estatística descritiva do factor Comportamentos Auto-referenciados por idades.

\begin{tabular}{lcccc} 
& Idade & N & Média & $\begin{array}{c}\text { Desvio } \\
\text { Padrão }\end{array}$ \\
\cline { 2 - 5 } & 12 & 30 & 32.90 & 3.89 \\
& 13 & 25 & 33.60 & 4.30 \\
Comport. & 14 & 29 & 32.72 & 3.14 \\
Auto- & 15 & 31 & 34.00 & 3.75 \\
-referenc. & 16 & 22 & 35.00 & 3.07 \\
& 17 & 9 & 31.11 & 3.41 \\
& 18 & 10 & 31.50 & 1.78 \\
\hline
\end{tabular}

Para o factor avaliação dos pais, os jovens com 12,13 e 17 anos de idade, apresentaram os valores mais elevados, respectivamente (quadro 12).

Quadro 12: Estatística descritiva do factor Avaliação dos Pais por idades.

\begin{tabular}{lcccc} 
& Idade & N & Média & $\begin{array}{c}\text { Desvio } \\
\text { Padrão }\end{array}$ \\
\cline { 2 - 5 } & 12 & 30 & 11.37 & 1.92 \\
& 13 & 25 & 10.72 & 2.26 \\
Avaliação & 14 & 29 & 9.45 & 2.23 \\
dos Pais & 15 & 31 & 10.03 & 2.75 \\
& 16 & 22 & 8.95 & 2.55 \\
& 17 & 9 & 10.78 & 2.39 \\
& 18 & 10 & 8.80 & 1.93 \\
\hline
\end{tabular}

Para o factor negativismo pré-competitivo, os valores médios mais elevados foram os dos jovens de 16, 14 e 17 anos, respectivamente (quadro 13).

Quadro 13: Estatística descritiva do factor Negativismo Pré-competitivo por idades.

\begin{tabular}{lcccc} 
& Idade & N & Média & $\begin{array}{c}\text { Desvio } \\
\text { Padrão }\end{array}$ \\
\cline { 2 - 5 } & 12 & 30 & 9.37 & 2.62 \\
& 13 & 25 & 8.56 & 2.74 \\
Negat. & 14 & 29 & 10.14 & 2.47 \\
Pré- & 15 & 31 & 9.68 & 2.80 \\
-compet. & 16 & 22 & 10.41 & 2.79 \\
& 17 & 9 & 10.00 & 2.24 \\
& 18 & 10 & 9.40 & 3.60 \\
\hline
\end{tabular}


A relação entre a percepção de competência física, índice de massa corporal e competência efectiva em jovens praticantes de basquetebol

Sandro Ferreira, Hélder Fernandes, José Vasconcelos-Raposo

$\mathrm{Na}$ atracção pelo Desporto, o valor médio mais alto é dos jovens de 14 anos, seguido dos jovens de 15 e 17 anos, respectivamente (quadro 14).

Quadro 14: Estatística descritiva do factor Atracção pelo Desporto por idades.

\begin{tabular}{lcccc} 
& Idade & N & Média & $\begin{array}{c}\text { Desvio } \\
\text { Padrão }\end{array}$ \\
\cline { 2 - 5 } & 12 & 30 & 8.63 & 1.65 \\
& 13 & 25 & 8.28 & 1.62 \\
Atracção & 14 & 29 & 9.00 & 1.13 \\
pelo & 15 & 31 & 8.81 & 1.35 \\
Desporto & 16 & 22 & 8.50 & 1.47 \\
& 17 & 9 & 8.78 & 1.09 \\
& 18 & 10 & 8.00 & 1.41 \\
\hline
\end{tabular}

Finalmente, para a facilidade de aprender habilidades, o valor médio mais elevado, verifica-se nos jovens com 17 anos, seguido dos jovens de 15 e 12 anos de idade, respectivamente (quadro 15).

Quadro 15: Estatística descritiva do factor Facilidade em aprender habilidades por idades.

\begin{tabular}{lcccc} 
& Idade & N & Média & $\begin{array}{c}\text { Desvio } \\
\text { Padrão }\end{array}$ \\
\cline { 2 - 5 } & 12 & 30 & 10.33 & 1.83 \\
& 13 & 25 & 10.12 & 1.81 \\
Fac. em & 14 & 29 & 10.00 & 2.04 \\
aprender & 15 & 31 & 10.61 & 2.04 \\
habilid. & 16 & 22 & 10.09 & 1.95 \\
& 17 & 9 & 11.00 & 1.00 \\
& 18 & 10 & 9.90 & 1.85 \\
\hline
\end{tabular}

\section{Discussão}

A discussão dos dados obtidos terá a seguinte sequência: (i) discussão dos resultados do IMC; (ii) discussão dos resultados da PCF e da CEB; e, (iii) discussão dos resultados obtidos no PCIS.
Segundo Barata ${ }^{4}$, as consequências nefastas dum défice ou de uma sobrecarga ponderal, têm conduzido a múltiplas tentativas de elaborar tabelas de pesos recomendáveis, com base na idade, altura e peso. A generalização dos seguros de vida e de saúde, implementou o uso de fórmulas deste tipo e algumas delas ainda em uso, são provenientes de importantes companhias de seguros norte-americanas. A crítica global a estas fórmulas é que não há um peso ideal em termos rígidos, mas sim um balizamento entre pesos aconselháveis, os quais variam em função do somatótipo do indivíduo. O IMC permite um escalonamento e tem várias vantagens sobre essas equações. Quando analisamos os resultados obtidos, quer nos rapazes (20.99 \pm 3.34$)$ quer nas raparigas $(20.73 \pm 2.90)$ e mesmo até nos grupos etários, verificamos que todos os valores estão dentro dos valores recomendáveis para a classificação de peso óptimo, que é entre 20.1 e $25 \mathrm{~kg} / \mathrm{m}^{2}$. Curiosamente, os valores apresentados pelos rapazes são superiores ao das raparigas.

Estes resultados são passíveis de várias interpretações. Como o IMC representa o quociente entre o peso e o quadrado da altura, e como a altura da população tem vindo a aumentar, é notório que o crescimento dos rapazes tem sido mais acentuado nos rapazes, do que nas raparigas. Outra interpretação que podemos retirar destes resultados, é que os rapazes têm vindo a aumentar de peso. Este aumento, por sua vez, pode ser devido ao aumento da massa muscular ou da massa gorda (MG). Barata refere que os jovens do sexo masculino são considerados obesos, quando a MG ultrapassa os 20 ou $25 \%$ do seu peso. Nos jovens do sexo feminino, considera-se haver obesidade quando os valores de MG atingem os 30\% do seu peso. Por sua vez, a evolução da sociedade e a par desta, a industrialização e a mecanização das tarefas dos jovens, tem provocado algumas alterações nos padrões de vida, o que tem resultado numa diminuição da quantidade de actividade, ou seja, uma hipo-actividade ${ }^{14}$. Esta pode muito bem ser 
a explicação para os valores de IMC superior nos rapazes. Também com esta modernização, os jovens começaram a ocupar grande parte do seu tempo a ver televisão e a brincar com os computadores. Os hábitos alimentares têm sofrido igualmente algumas alterações na população em geral, e também pode ter um efeito no aumento de peso da população.

Não encontramos diferenças estatisticamente significativas entre sexos, mas sim entre idades $(\mathrm{F}=4.031 ; \mathrm{p}<0.046)$. Isto significa, que o IMC tem um crescimento natural em cada faixa etária, devido aos processos maturacionais que os jovens vão sofrendo.

Horn e Weiss ${ }^{38}$ correlacionaram a percepção $^{2}$ de competência (PC) com a competência efectiva (CE), medida por professores e estes dados revelaram que os jovens tornaram-se mais precisos na sua PC com a idade. Este estudo também correlaciona a PCF com a CE. No entanto, na CE utilizamos os LL do Basquetebol, porque desta forma não tivemos nenhuma interferência para a obtenção desta variável. A correlação foi fraca mas significativa $(r=0.182$; $\mathrm{p}<0.05)$, pelo que podemos afirmar que a amostra é precisa na sua PCF. Estes autores referem que estas diferenças na precisão de análise da PC podem estar associadas, pelo menos em parte, às fontes de informação que os jovens usam para julgar a sua competência.

No presente estudo, os rapazes obtiveram valores de PCF superiores aos das raparigas (25.01 \pm 3.96 e $22.00 \pm 3.79$, respectivamente). Os rapazes obtiveram igualmente valores superiores de CEB. Estes resultados vêm corroborar que a PCF dos rapazes é bastante precisa. Williams e Gill $^{13}$ também encontraram valores significativamente mais elevados de PCF nos rapazes do que nas raparigas $(F=15.15 ; \mathrm{p}<0.01)$. Nos estudos de Klint e Weiss ${ }^{40}$ e Ryckman e Hamel ${ }^{55}$, os jovens com uma elevada PCF estavam mais motivados para desenvolver as suas habilidades, ao contrário dos seus colegas. E ainda consideravam o divertimento como uma razão para a sua prática, ou seja, reportaram factores intrínsecos como mais importantes que os factores extrínsecos.

Feltz e Petlichkoff ${ }^{21}$ relacionaram a PCF com a participação dos jovens nos programas desportivos. Estes autores encontraram valores de PCF mais elevados nos jovens que participavam no Desporto, relativamente aos jovens que desistiram dessa participação. Nesse mesmo estudo, os rapazes também obtiveram resultados superiores às raparigas na PCF $(2.97 \pm 0.51 \mathrm{e}$ $2.75 \pm 0.49$, respectivamente). Resultados idênticos obtiveram-se na nossa amostra, em que os rapazes tiveram valores médios de atracção pelo Desporto $(8.89 \pm 1.41)$ superiores às raparigas (8.38 \pm 1.41$)$, chegando-se mesmo a verificar diferenças estatisticamente significativas $(\mathrm{F}=5.065$; $\mathrm{p}<0.026)$. Podemos inferir que provavelmente, os rapazes da nossa amostra têm uma maior predisposição para a participação em programas desportivos. E isto porque, Weiss e Duncan ${ }^{64}$ referem que existe uma relação entre a competência física e a aceitação social $(\mathrm{r}=0.75$; $\mathrm{p}<0.05$ ), sugerindo que os jovens acreditam que as habilidades atléticas estão totalmente associadas à percepção de aceitação social. Também Roberts ${ }^{54}$ encontraram uma evidência que sugere que uma elevada PCF levaria os jovens a esperar mais sucesso desportivo no futuro e desta forma, prolongarem o seu envolvimento nesse Desporto.

Quando analisamos a PCF e a CEB nos escalões etários, comprovamos que nem todos os jovens com uma elevada PCF (por exemplo os jovens de 18 anos, apresentam um valor médio de $23.30 \pm 2.58)$ são na prática, competentes no domínio da CEB (17.00 \pm 15.67 , respectivamente). No entanto, os jovens de 12 anos que possuíam valores médios de PCF de 25.20 \pm 3.60 , são de facto, dos mais competentes no domínio físico da modalidade de Basquetebol (24.33 \pm 16.54 , respectivamente). Concomitantemente, o contrário também se verificou. Assim, os jovens com 16 anos obtiveram os valores mais baixos de PCF $(22.27 \pm 4.61)$ e no entanto, foram aqueles que 
conseguiram ser mais competentes nesta modalidade (25.45 18.19$)$. A análise multivariada revelou-nos diferenças estatisticamente significativas para a idade, no domínio da PCF $(\mathrm{F}=4.031$; $\mathrm{p}<0.046)$. Estes resultados podem reflectir o crescente interesse dos jovens por outras actividades que não sejam as desportivas. Os jovens de 12 anos, ainda brincam na rua e socialmente não lhes fica mal, jogarem à bola ou correrem nos pátios das escolas. Para os jovens de 16 anos, embora os resultados demonstramse competentes nesta modalidade, estes não se percepcionam como tal. Isto acontece, devido a um período de grandes transformações esqueléticas, o que pode tornar o jovem "desajeitado". Assim, o desenvolvimento da estatura é mais acentuado que o do peso. E aumenta o interesse pela aparência pessoal, porque também aumenta $\mathrm{o}$ interesse pelo sexo oposto.

O modelo da competência motivacional de Harter $^{28,30}$ ajudará os educadores a compreenderem e a explicarem o comportamento dos jovens e dos adolescentes. As relações entre faixas etárias e as fontes de informação que os jovens utilizam para construir a sua PC, foram novamente aqui estudadas.

Assim como os dados de Horn e Hasbrook ${ }^{35}$ e Horn e Weiss ${ }^{38}$, neste estudo os jovens mais novos (12 anos) obtiveram valores médios de avaliação dos pais (à excepção dos jovens de 17 anos), superiores aos jovens mais velhos $(>15$ anos). O mesmo não sucede nos valores de comparação social. Os resultados de Horn e Hasbrook, assim como com o de Horn e Weiss) e Weiss, Horn e Ebbeck ${ }^{70}$ encontraram valores superiores na comparação social nos jovens mais velhos, comparativamente aos jovens mais novos. O nosso estudo, apresenta valores de comparação social superiores nos jovens mais novos. Os jovens de 12 anos, apresentaram valores de 43.00 \pm 6.72 e os jovens de 13 anos apresentaram um valor de $43.36 \pm 7.13$. Estes autores referem que há um padrão comum nestes jovens (os jovens da sua amostra são bastante mais novos: 8-13 anos). Ambas as faixas etárias apresentam valores elevados de negativismo pré-competitivo e valores baixos de PC. Os jovens mais novos do nosso estudo (12-13 anos) são os que apresentam os valores mais elevados de PCF (25.20 \pm 3.60 e $24.44 \pm 4.50$, respectivamente) e são os que apresentam os valores mais baixos de negativismo pré-competitivo $(9.37 \pm 2.62$ e $8.56 \pm 2.74$, respectivamente).

Horn e Hasbrook ${ }^{35}$ estudaram em dois grupos etários, o primeiro com uma média de idades de 9.07 anos e o segundo com 12.81 anos, a PCF e as respectivas fontes de informação que definem essa mesma competência. Para a nossa discussão, podemos utilizar este segundo grupo. Especificamente, os jovens com 10-13 anos que tinham uma percepção de competência baixa e elevados índices de negativismo pré-competitivo, apresentaram a comparação social como fonte preferencial para a construção da competência. Neste estudo, são os jovens de 12-13 anos que apresentam os valores médios mais elevados na comparação social, mas são os que apresentam os valores médios mais baixos na negativismo pré-competitivo e valores mais altos na PCF. Com o aumento da idade, os critérios internos (por exemplo, os comportamentos auto-referenciados) tendem a prevalecer. No entanto, este efeito pode ser alterado, quando os jovens possuem elevados índices de negativismo précompetitivo e baixos índices de $\mathrm{PC}^{70}$. Tanto as influências ambientais como as estratégias de controlo individual, providenciam fontes de informação da competência física, a partir das quais os jovens podem aprender a divertir-se com as experiências e sentirem-se bem com eles mesmos $^{66}$. O Desporto permite a comparação social, muitas vezes à custa de objectivos individuais de cada um. Ames ${ }^{2}$ e Butler ${ }^{10,11}$ referem que a adopção de objectivos auto-referenciados, poderá encaminhar os indivíduos para a aprendizagem e para sua a melhoria sucessiva. 
Os treinadores e professores de Educação Física podem ser importantes fontes de informação de competência, através do feedback correctivo e do elogio ${ }^{5,37,56}$. Mais especificamente, Smoll et al. ${ }^{56}$ verificou que os treinadores que davam mais elogios e feedbacks correctivos tiveram como efeito, o aumento da auto-estima dos jovens, que por sua vez, apresentavam índices baixos de auto-estima no início da época. Allen e Howe $^{1}$, revelaram que o feedback do treinador contribui significativamente para a explicação de uma elevada percepção de competência de atletas de hóquei em gelo $(\mathrm{F}=3.24 ; \mathrm{p}<0.05)$. Thill e Brunnel 59 encontraram valores de PCF elevados, após feedbacks positivos, em detrimento de feedbacks negativos $(\mathrm{F}=4.426$; $\mathrm{p}<0.05)$.

Segundo Weiss ${ }^{66}$, a teoria da competência motivacional de Harter, bem como a investigação efectuada neste domínio, sugerem que o feedback quantitativo e qualitativo, assim como o reforço de outros, influenciam a percepção de competência dos jovens. O feedback que o jovem recebe da prática desportiva, fornece um conjunto de informações que reflectem a representam as suas capacidades e utilidade. É especialmente verdade, para os jovens do $1 .^{\circ}$ Ciclo e para os que têm uma percepção errada ou baixa da sua competência efectiva, que parecem depender mais da avaliação social, que os jovens mais velhos. No que respeita à avaliação dos adultos (por exemplo, os pais), como fonte de informação para o julgamento da competência dos jovens, os profissionais da Educação Física e Desporto, deverão tomar em atenção a quantidade absoluta e relativa de reforço que dão aos jovens, a contingência do reforço que conduz ao comportamento e à responsabilidade do sucesso, assim como os erros das habilidades. No que concerne ao número e contingência do reforço, Horn $^{39}$ resume os estudos que encontrou e refere que demasiados elogios quando há sucesso numa tarefa fácil ou numa prestação medíocre, pode provocar uma fraca expectativa do jovem. Isto é, por sua vez, leva a um decréscimo da PC.
Igualmente, críticas sucessivas na forma de feedbacks correctivos, podem levar os jovens a pensarem em elevadas expectativas no futuro. Assim, os treinadores estão mais predispostos a reforçar os jovens mais fracos e a criticar os que têm mais potencial, e que se espera mais sucesso no futuro.

Para Weiss ${ }^{66}$, uma estratégia apropriada pode ser o que se designa de "desafios ideais". Um "desafio ideal" é aquele que adequa a actividade ao jovem e não o jovem à actividade. Os jovens e adolescentes sentem uma grande satisfação quando conseguem alcançar sucesso nas actividades que realizam, por outras palavras, sentem satisfação quando o êxito deriva da adaptação das actividades às suas próprias capacidades. As actividades que permitem definir esses "desafios ideais" têm que respeitar: (i) uma sequência lógica de construção e instrução; (ii) focar os aspectos de aprendizagem em vez de resultados; e, (iii) permitir um estilo de ensino indirecto.

Horn e Hasbrook ${ }^{36}$, referem que os jovens com uma elevada PC, identificam os critérios internos e a comparação com os amigos, como fontes de informação para julgar a sua competência. Os nossos resultados, indicam que os rapazes tendem a compararem-se socialmente, mais que as raparigas e a atraírem-se mais pelas actividades desportivas. Os mesmos autores referem ainda, que os jovens com uma baixa PC identificam os elogios e reforços dos pais, como fonte de informação para julgar a sua competência. Neste estudo, a avaliação dos pais obteve valores médios semelhantes para os rapazes $(10.22 \pm 2.54)$ e raparigas $(9.99 \pm 2.46)$.

Os resultados de Williams ${ }^{74}$ indicam uma relação significativa entre a orientação cognitiva e as fontes de informação que constroem a PC. A orientação cognitiva para a tarefa, está relacionada com: (i) o aprender e melhorar; (ii) os comentários dos pais; (iii) o grau de divertimento; e, (iv) a atitude antes do jogo. Por sua vez, a orientação cognitiva para o ego, está relacionada com: (i) comparação com os colegas; 
(ii) o aprender e o melhorar; e, (iii) os comentários dos pais. Dweck e Elliot ${ }^{19}$ afirmam que os jovens que estão orientados para o resultado, estão interessados em receber julgamentos favoráveis relativos à sua competência e evitar receber julgamentos negativos da sua competência, vindo de outros. Assim, os objectivos da busca da aprendizagem (orientação para a tarefa) e de obter um resultado (orientação para o ego), levam os jovens a processar a informação acerca da sua prestação de forma diferente. Os jovens que estão orientados para aprender, fazem questões do tipo: "Como é que eu chego lá?", "O que é que eu vou aprender?", enquanto que os que estão orientados para o resultado, fazem questões do tipo: "Será que posso fazer aquilo?" ou "Será que pareço bem no final disto?".

A partir do nosso estudo, podemos retirar as seguintes conclusões:

verificaram-se diferenças estatisticamente significativas entre sexos para o IMC $(\mathrm{F}=4.031 ; \mathrm{p}<0.046)$, para a PCF $(\mathrm{F}=21.216$; $\mathrm{p}<0.000)$ e para a CEB (24.484; $\mathrm{p}<0.000)$;

no IMC, a média dos rapazes $(20.99 \pm 3.34)$ foi superior à das raparigas $(20.73 \pm 2.90)$;

na PCF, a média dos rapazes (25.01 \pm 3.96$)$ foi superior à das raparigas $(22.00 \pm 3.79)$;

- na CEB, a média dos rapazes $(25.13 \pm 16.85)$ foi superior à das raparigas (13.50 111.26$)$; verificaram-se diferenças estatisticamente significativas entre sexos para os seguintes factores do PCIS: negativismo pré-competitivo $(\mathrm{F}=6.522 ; \mathrm{p}<0.012)$ e atracção pelo Desporto $(\mathrm{F}=5.065$; $\mathrm{p}<0.026)$; e,

verificaram-se diferenças estatisticamente significativas entre as faixas etárias, para o factor avaliação dos pais $(\mathrm{F}=11.151 ; \mathrm{p}<0.001)$.

\section{Correspondência}

José Vasconcelos-Raposo

Rua Dr. Manuel Cardona

5000-558 Vila Real. Portugal

E-mail: j.vasconcelos.raposo@gmail.com

\section{Referências}

1. Allen, J. B. e Howe, B. L. (1998). Player ability, coach feedback and female adolescent athletes' perceived competence and satisfaction. Inter-national Journal of Sport Psychology, 20, 280299.

2. Ames, C. (1992). Achievement goals, motivational climate and motivational processes. In G.C. Roberts (Ed.), Motivation in Sport and Exercise (pp. 161-176). Champaign: Human Kinetics.

3. Bandura, A. (1977). Self-efficacy: Toward a unifying theory of behavioural change. Psychological Review, 84, 191-215.

4. Barata, T. (1997). Actividade Física e Medicina Moderna. Lisboa: Europress.

5. Black, S. J. e Weiss, M. R. (1992). The relationship among perceived coaching behaviours, perceptions of ability and motivation in competitive age-group swimmers. Journal of Sport \& Exercise Psychology, 14, 309-325.

6. Block, J. H. e Block, J. (1980). The role of ego-control and ego-resiliency in the organization of behaviour. In W.A. Collins (Ed.), Development of cognition, affect and social relations (pp. 39-101). Hillsdale: Erlbaum.

7. Butler, R. (1989). Interest in task and interest en peers' work in competitive and non-competitive conditions: A development study. Child Development, 60, 562-570.

8. Butler, R. (1990). Mastery versus ability appraisal: A development study of children's observations of peers' work. Child Development, 61, 201-210.

9. Butt, D. S. (1987). Psychology of Sport: The behaviour, motivation, personality and performance of atbletes. New York: Van Nostrand Reinhold Company.

10. Charlesworth, W. R. (1979). Ethology: Understanding the other half of intelligence. In M. Von Cranacha; K. Foppa; W. Lepenies e D. Ploog (Eds.), Human ethology: Claims and limits of a new discipline (pp-491-519). Cambridge: Cambridge University Press. 
11. Costa, D. (1997). A influência da actividade física nos níveis de saúde, condição física e hábitos de saúde. Horizonte, 77 (dossier), 1-8.

12. Duda, J. L.; Chi, L.; Newton, M. L.; Walling, M. D. e Catley, D. (1995). Task and ego orientation and intrinsic motivation in sport. International Journal of Sport Psychology, 26, 40-63.

13. Dweck, C. S. e Elliot, E. S. (1984). Achievement motivation. In M. Hetherington (Ed.), Social development: Carmichael's manual in child psychology (pp. 643-691). New York: Wiley.

14. Dweck, C. S. (1985). Intrinsic motivation, perceived control and self-evaluation maintenance: An achievement goal analysis. In C. Ames e R. Ames (Eds.), Research on motivation in education: The classroom milieu (vol. 2, pp. 289-305). Orlando: Academic Press.

15. Feltz, D. e Petlichkoff, L. (1983). Perceived competence among interscholastic participants and dropout. Canadian Journal of Applied Sport Sciences, 8, 231-235.

16. Ford, M. E. (1985). The concept of competence. Themes and variations. In H.A. Marlowe Jr. e R.B. Weinberg. (Eds.), Competence development: Theory and practice in special populations (pp. 3-49). Springfield: Charles C. Thomas.

17. Garmezy, N. e Masten, A. S. (1991). The protective role of competence indicators in children at risk. In E. M. Cummings; A. L. Greene e K. H. Karrakar (Eds.), Life span development psychology: Perspectives on stress and coping (pp. 151-174). Hillsdale: Erlbaum.

18. Garmezy, N. (1970). Process and reactive schizophrenia: Some conceptions and issues. Schizophrenia Bulletin, 2, 30-70.

19. Harter, S. e Connel, A. (1984). A model of children's achievement and related self-perceptions of competence, control and motivational orientation. In J. Nicholls (Ed.), Development of achievement motivation (vol. 3). Greenwich: JAI Press.

20. Harter, S. (1978). Effectance motivation reconsidered. Human Development, 21, 34-64.

21. Harter, S. (1981). A new self-report scale of intrinsic versus extrinsic orientation in the classroom: Motivational and informational components. Development Psychology, 17, 300-312.

22. Harter, S. (1982). The perceived competence scale for children. Child Development, 53, 87-97.

23. Hartman, H. (1958). Ego psychology and the problem of adaptation. New York: International Universities Press.

24. Horn, T. S. e Hasbrook, C. A. (1986). Informational components influencing children's perceptions of their physical competence. In M. R. Weiss e D. Gould (Eds.), Sport for children and youth (pp. 81-88). Champaign: Human Kinetics.

25. Horn, T. S. e Hasbrook, C. A. (1987). Psychological characteristics and the criteria children use for self-evaluation. Journal of Sport Psychology, 9, 208-221.

26. performance (pp. 68-81). Palo Alto: Mayfield.

27. Horn, T. S. e Weiss, M. R. (1991). A development analysis of children's self-ability judgments in the physical domain. Pediatric Exercise Science, 3, 310-326.

28. Horn, T. S. (1985). Coaches feedback and changes in the children's perceptions of their physical competence. Journal of Educational Psycho$\log y, 77,174-186$.

29. Klint, K. A. e Weiss, M. R. (1987). Perceived competence and motives for participating in youth sports: A test of Harter's competence motivational theory. Journal of Sport Psychology, 9, 55-65.

30. Klint, K. A. (1985). Participation motives and self-perceptions of current and former atbletes in youth gymnastic. Unpublished master's thesis. University of Oregon, Eugene.

31. Loevinger, J. (1976). Ego Development. San Francisco: Jossey-Bass.

32. Malina, R. M. e Bouchard, C. (1986). Growth, maturation and physical activity. Champaign: Human Kinetics.

33. Masten, A. S.; Coatsworth, J. D.; Neeman, J.; Gest, S. D.; Tellegen, A. e Garmezy, N. (1995). The structure and coherence of competence from childhood through adolescence. Child Development, 66, 1635-1659. 
A relação entre a percepção de competência física, índice de massa corporal e competência efectiva em jovens praticantes de basquetebol

Sandro Ferreira, Hélder Fernandes, José Vasconcelos-Raposo

34. Newton, M. e Duda, J. L. (1999). The interaction of motivational climate, dispositional goal orientations and perceived ability in predicting indices of motivation. International Journal of Sport Psychology, 30, 63-82.

35. Nicholls, J. G. (1978). The development of concepts of effort and ability, perceptions of own attainment and the understanding that difficult tasks require more ability. Child Development, 49, 800-814.

36. Nicholls, J. G. (1989). The competitive ethos and democratic education. Cambridge: Harvard University Press.

37. Phillips, L. (1968). Human adaptation and its failures. New York: Academic Press.

38. Roberts, G. C. (1984). Toward a new theory of motivation in sport: The role of perceived ability. In J. Silva e R. S. Weinberg (Eds.), Psychological Foundations of Sport. Champaign: Human Kinetics.

39. Roberts, G. C.; Kleiber, D. A. e Duda, J. L. (1981). An analysis of motivation in children's sport: The role of perceived competence in participation. Journal of Sport Psychology, 3, 206-216.

40. Ryckman, R. M. e Hamel, J. (1993). Perceived physical ability differences in the sport participation motives of young athletes. International Journal of Sport Psychology, 24, 270-283.

41. Smoll, F. L.; Smith, R. E.; Barnett, N. P. e Everett, J. J. (1993). Enhancement of children's self-esteem through social support training for youth sport coaches. Journal of Applied Psychology, 78, 602-610.

42. Sroufe, L. A. (1979). The coherence of individual development: Early care attachment and subsequent development issues. American Psychologist, 34, 834-841.

43. Sternberg, R. J. (1985). Beyond IQ: A triarcbic theory of human intelligence. Cambridge: Cambridge Academic Press.

44. Thill, E. E. e Brunnel, P. (1995). Ego-involvement and task-involvement: Related conceptions of ability, effort and learning strategies among soccer players. International Journal of Sport Psychology, 26, 81-97.
45. Water, E. e Sroufe, L. A. (1983). Social competence as a development construct. Development Review, 3, 79-97.

46. Wechsler, D. (1958). The measurement and appraisal of adult intelligence. Baltimore: Williams and Wilkins.

47. Weigang, D. A. e Broadhurst, C. J. (1998). The relationship among perceived competence, intrinsic motivation and control perceptions in youth soccer. International Journal of Sport Psycho$\log y, 29,324-338$.

48. Weiss, M. R. e Chaumeton, N. (1992). Motivational orientations in sport. In T. S. Horn (Ed.), Advances in Sport Psychology. Champaign: Human Kinetics.

49. Weiss, M. R. e Duncan, S. C. (1992). The relationship between physical competence and peer acceptance in the context of children's sport participation. Journal of Sport \& Exercise Psychology, 14, 177-191.

50. Weiss, M. R. e Horn, T. S. (1990). The relation between children's accuracy estimates of their physical competence and achievement-related characteristics. Research Quarterly for Exercise \& Sport, 61, 250-258.

51. Weiss, M. R. (1987). Self-esteem and achievement in children's sport and physical activity. Advances in Pediatric Sport Sciences, 2, 87-120.

52. Weiss, M. R.; Horn, T. S. e Ebbeck, V. (1997). Children's self-perceptions and sources of physical competence information: A cluster analysis. Journal of Sport \& Exercise Psychology, 19, 52-70.

53. White, R. (1959). Motivation reconsidered: The concept of competence. Psychological Review, 66, 297-333.

54. Williams, L. e Gill, D. L. (1995). The role of perceived competence in the motivation of physical activity. Journal of Sport \& Exercise Psychology, 17, 363-378.

55. Williams, L. (1994). Goal orientation and athletes' preferences for competence information sources. Journal of Sport \& Exercise Psychology, 16, 416-430.

56. Zigler, E. e Glick, M. (1986). A development approach to adult psychopathology. New York: Wiley. 